\title{
El debate sobre la cuestión urbana y el Trabajo Social: contribuciones críticas para pensar la profesión en territorios de relegación urbana ${ }^{1}$
}

\author{
Víctor Orellana ${ }^{2}$ \\ Alexander Panez ${ }^{3}$
}

\section{RESUMEN}

El Trabajo Social que debate y actúa sobre territorios de relegación urbana posee hoy el desafío de apropiarse de teorías críticas que le permitan elaborar herramientas conceptuales y metodológicas a través de las cuales describir complejamente dichos territorios, proponer procesos de intervención social que tornen efectivos los derechos de sus habitantes, como también confrontar los discursos afines al proyecto neoliberal que predominan en la orientación de las políticas sociales. En este texto se presentan algunas de esas teorías y, luego, se rastrea su presencia en nuestra formación profesional.

Palabras clave: Trabajo social, ciudad, espacio, territorios de relegación urbana, marginalidad avanzada

\section{O debate sobre a questão urbana e o Serviço Social: contribuições críticas para pensar na profissão em territórios de relegação urbana.}

\section{RESUMO}

O Serviço Social que debate e atua em territórios de relegação urbana possui hoje o desafio de se apropriar de teorias críticas que lhe permitam elaborar

Artículo recibido: 03/11/2015. Artículo aprobado: 19/07/2016.

Este trabajo es una versión ampliada y corregida de la ponencia titulada "Trabajo social desde la ciudad marginada", presentada por los autores en el XXI Seminario Latinoamericano de Escuelas de Trabajo Social. La formación profesional en Trabajo Social: avances y tensiones en el contexto de América Latina y el Caribe. "A 50 años del movimiento de Reconceptualización”. Universidad Autónoma de Sinaloa. Mazatlán, México. 28-30 de setiembre y 1 de octubre 2015.

2 Chileno. Trabajador social. Doutorando do Programa de Pós-graduação em Serviço Social-Universidade Estadual do Rio de Janeiro, Brasil.

E-mail: v.orellanabravo@gmail.com.

3 Chileno. Trabajador social. Doutorando do Programa de Pós-graduação em Geografia-Universidade Federal Fluminense, Rio de Janeiro, Brasil. E-mail: alexander.panez@gmail.com. 
ferramentas conceptuais e metodológicas através das quais descrever complexamente esses territórios, propor processos de intervenção social que avancem na efetivação dos direitos dos seus moradores desses, como também, confrontar os discursos próximos ao projeto neoliberal que predominam na orientação das políticas sociais. No texto apresentam-se algumas de essas teorias e, logo depois, rastreia-se a presencia dessas perspectivas em nossa formação profissional.

Palavras chave: Serviço Social; Cidade; Espaço; Territórios de relegação urbana; Marginalidade avançada.

\title{
The debate over the urban issue and the Social Work: contributions for thinking the profession on territories of urban relegation.
}

\author{
ABSTRACT:
}

Nowadays, the Social Work that discusses and acts on territories of urban relegation has the challenge of incorporate critical theories that might allow to develop conceptual and methodological tools to (i) describe with more complexity those territories, (ii) propose social intervention in order to become effective the rights of the people that live in these zones of the city, and also (iii) confront the speeches that are close to the neoliberal project and are predominant in the orientation of the social policies. In this article are presented some of these theories and also we research their presence in our professional training.

Keywords: Social Work; City; Space; Territories of urban relegation; Advanced marginality.

\section{Introducción}

Uno de los mayores desafíos que vive el Asistente Social en el presente es desarrollar su capacidad de descifrar la realidad y construir propuestas de trabajo creativas y capaces de tornar efectivos los derechos (...) ir más allá de las rutinas profesionales y buscar aprehender el movimiento de lo real para detectar tendencias y posibilidades factibles de ser impulsadas (Iamamoto, 2003, p. 33).

Los/as trabajadores/as sociales somos, probablemente, los profesionales de las ciencias sociales que más tiempo destinamos hacia lo que se conoce como "trabajo en terreno", esa idea de "estar con la gente", escuchando, viendo y sintiendo in situ sus problemáti- 
cas. Cuando el área de intervención se sitúa en las periferias de las grandes ciudades, el/la asistente social ${ }^{4}$ se vuelca radicalmente sobre un trabajo en la calle, se vincula con las dinámicas locales, incluso ingresa a los hogares de las familias, se involucra con sus vidas e intenta desenvolver su trabajo en una cotidianidad donde se dan complejos fenómenos sociales.

Por otro lado, la problematización teórica que se realiza para abordar fenómenos sociales vinculados a las periferias urbanas ha incorporado, de manera creciente y sostenida en los últimos años, elementos conceptuales que se inscriben en la tradición del debate sobre la cuestión urbana. Así, la discusión de conceptos como territorio y espacio, los abordajes para pensar la ciudad, entre otros, se han instalado con fuerza en el vocabulario oficial de nuestras políticas y programas sociales durante las últimas décadas.

En este contexto, el interés de este trabajo es poder realizar una aproximación exploratoria que nos permita caracterizar la relación que tenemos desde la formación y ejercicio profesional del Trabajo Social con dicho debate. Así, las inquietudes que motivan el presente escrito podrían traducirse en las siguientes preguntas:

¿Cuál es el referencial teórico con el que trabajadores/as sociales enfrentamos fenómenos sociales expresivos de la cuestión urbana contemporánea en los procesos de intervención social en los que nos desempeñamos? ¿Qué conceptos de ciudad y qué lecturas acerca de su producción y transformación en tanto espacio orientan la acción profesional?

\footnotetext{
En el contexto latinoamericano, la denominación profesional varía según el país, conteniendo tanto a asistentes sociales como también trabajadores/as sociales, al mismo tiempo que en los diferentes países nos encontramos con Trabajo Social y Servicio Social. Dichas distinciones poseen un profuso debate -que no abordaremos en el presente texto- que determina uno u otro término, los cuales serán utilizados indistintamente a lo largo del texto.
} 
Planteamos estas preguntas no para agotar posibles respuestas, sino para provocar una discusión que nos parece central en un presente donde, como veremos más adelante, el capitalismo avanza avasalladoramente en el ordenamiento de la inmensa mayoría de nuestras ciudades latinoamericanas, donde la población urbana crece precaria y sostenidamente año tras año.

En este escenario, se vuelve urgente para el Trabajo Social reflexionar sobre la pertinencia de sus categorías de abordaje hacia la cuestión urbana, sobre todo para una profesión que tradicionalmente, al menos en el caso de América Latina, ha tenido como categoría central de análisis el concepto de comunidad y, consecuentemente, el llamado Trabajo Social Comunitario como lugar teórico y éticopolítico de reflexión y acción.

Sustentamos, a modo de hipótesis, que el Trabajo Social que debate y actúa sobre fenómenos sociales expresivos de la cuestión urbana, padece hoy de un déficit de teorías críticas que le permitan fundamentar tanto su reflexión como su acción profesional en tales áreas de intervención. Lo anterior posee, al menos, dos consecuencias negativas.

En primer lugar, nos impide pensar modos de intervención que den cuenta de la totalidad al interior de la cual comprender los fenómenos sociales localmente situados, muchas veces aprehendidos como dramas aislados cuya génesis remite apenas a los propios territorios y sus habitantes. De allí se deriva una segunda consecuencia negativa, pues la ausencia de teorías críticas ha dejado abierta la puerta para que la discursividad liberal sea la que predomine casi sin contrapeso en la formulación de políticas sociales, traducida en sendos (y carísimos) programas sociales, tanto públicos como privados, espacios en los cuales desarrollamos nuestra acción profesional. Sofocados por la experticia conservadora, nos hacen faltas herramientas críticas para describir complejamente dichos territorios y proponer procesos de intervención social que garanticen el ejercicio de derechos de los millones de personas que habitan a la sombra de nuestras comodidades. 
Evidentemente que en un texto no es posible (ni deseable) cerrar el debate respecto de cuáles, consideramos, son las categorías críticas con las que deberíamos dialogar. Nuestro objetivo en este artículo es presentar algunos conceptos que nos parecen centrales para discutir, desde el Trabajo Social, la cuestión urbana actual. Para conseguir el objetivo propuesto, nos hemos planteado abordar, en una primera sección, el concepto de espacio como también una mirada sobre la situación actual de las ciudades latinoamericanas y algunos fenómenos que en su interior están sucediendo. Dentro de estos fenómenos, pondremos especial énfasis en lo que ocurre en sus periferias, donde se aglomeran los pobres urbanos. Para tal tarea, trabajaremos con las propuestas conceptuales que ofrece Löic Wacquant, a partir de las cuales comprendemos a las periferias urbanas como territorios de relegación urbana que se desarrollan al alero de procesos de marginalidad avanzada, en un contexto de desarrollo de la fase penal del Estado.

En una segunda sección, iremos a la búsqueda de los conceptos previamente discutidos en los registros de nuestra profesión a nivel latinoamericano. En ese sentido, se trata de un primer ejercicio a través del cual procuramos la presencia de dichos soportes conceptuales en el Trabajo/Servicio Social latinoamericano, a partir de dos unidades de análisis: los planes de estudio de las Escuelas de Trabajo/Servicio Social y la discusión, por la vía de ponencias, de dichas temáticas en los Congresos/Seminarios/Encuentros de la profesión durante la última década. Cabe resaltar que se trata exclusivamente de una búsqueda de la enunciación de cinco conceptos (marginalidad, urbano, ciudad, espacio, territorio) en los planes y ponencias que fueron revisadas y no un análisis y problematización conceptual, para lo cual serían necesarias otras pesquisas con otros niveles de profundidad.

Finalmente, cerramos el texto señalando los que consideramos son desafíos importantes tanto para la formación como para el ejercicio profesional del Trabajo/Servicio Social, así como también proyecciones de posibles caminos de pesquisa. 


\section{Algunos debates centrales}

\section{Hacia una apropiación crítica del espacio}

Si el espacio tiene un aspecto neutro... es precisamente porque ya está ocupado, acondicionado, porque ya es objeto de estrategias antiguas, de las que no siempre se consigue encontrar las huellas. El espacio ha sido formado, modelado, a partir de elementos históricos o naturales, pero siempre políticamente. El espacio es político e ideológico (Léfèbvre, 1976, p. 46).

Desde nuestra perspectiva, pensar la relación del Trabajo Social con las transformaciones de la cuestión urbana, requiere reflexionar sobre la concepción de espacio. Entender lo que ocurre en nuestras ciudades nos convida a otras aproximaciones. Ahora bien, ¿Por qué es relevante para el Trabajo Social analizar el concepto de espacio y cuestionarnos a partir de éste?

En primer lugar, es importante señalar que la relación del Trabajo Social con la reflexión espacial, tiene como contexto mayor el lugar que las ciencias sociales le han otorgado al espacio dentro de sus análisis. Históricamente, la relación entre espacio y ciencias sociales ha estado marcada por más desencuentros que encuentros, debido al predominio que durante gran parte del siglo XIX y $\mathrm{XX}$ han tenido en la teoría social, enfoques centrados en el estudio de las acciones sociales, estructuras y/o sistemas sociales sin relevar al espacio como reflexión para comprender los fenómenos sociales (Hiernaux y Lindón, 2008). Lo anterior, nos ha llevado a configurar ciencias sociales profundamente a espaciales en sus lecturas de la realidad; sin embargo, podemos apreciar en la actualidad "una serie de tendencias que buscan descentrar la temporalidad y re-centrar el espacio como categoría fundamental para entender lo social y lo real" (Escobar, 2013, p. 91).

Pero, ¿qué entendemos por espacio? Antes que todo, debemos reconocer que espacio es una categoría profundamente densa, largamente debatida en disciplinas como la Geografía y donde existen diversas (e incluso contrapuestas) perspectivas desde las que pue- 
de ser comprendido. En el marco de este debate, nos situaremos desde los aportes de la geografía crítica.

Caracterizando la categoría espacio, podemos ver que algo compartido en varias perspectivas que lo conceptualizan, es la definición de su carácter de totalidad. Esto es clave, porque nos señala que el espacio no se trata exclusivamente de una dimensión física o topográfica de la realidad en la que nos desenvolvemos, sino que remite a los objetos creados por el ser humano y las relaciones sociales entre éstos (los sistemas de objetos y sistemas de acciones que analiza Milton Santos [1994]), a lo que nosotros mencionaríamos para diferenciar los bienes comunes naturales (a diferencia de su inclusión dentro del sistema de objetos que realiza Milton Santos), los animales y otros seres vivos como parte constitutiva del espacio.

Comprender esta característica de totalidad, ya nos permite una primera contribución al debate profesional, la que tiene que ver con reconocer la importancia no sólo de las relaciones sociales, sino también, de la materialidad en los fenómenos en los que el Trabajo Social se desenvuelve, donde por materialidad entendemos, desde una vivienda social de $30 \mathrm{~m}^{3}$ relegada a la periferia de la ciudad (pasando por pasajes, calles y plazas), hasta llegar a cerros, valles, ríos y ex-ríos (como ocurre en varios lugares del continente azotados por el despojo hídrico).

A partir de allí, podemos complejizar nuestra mirada sobre los fenómenos sociales al relevar la inseparabilidad de las relaciones sociales con el espacio construido y apropiado como naturaleza aspecto que no ha contado con el mismo desarrollo teórico que el análisis de las relaciones sociales en el Trabajo Social-. El reconocimiento de esta condición nos permite desmitificar algunas miradas sobre el espacio, como aquellas concepciones que suelen entenderlo como algo inmóvil, inmutable, como un cierto contenedor de las relaciones sociales. Si reconocemos las relaciones sociales como parte constitutiva del espacio, cabe preguntarnos: ¿cómo se desenvuelven estas relaciones y cómo afectan (y son afectadas) por la dinámica espacial? 
Observando nuestra realidad actual, vemos relaciones sociales marcadas por profundas desigualdades que separan, relegan, invisibilizan, violentan y oprimen a parte importante de los seres humanos que habitamos el planeta. He aquí una contradicción fundamental de nuestro ser en el espacio: si bien todos/as deambulamos por éste (algunos con mayor capacidad de movilidad que otros), muchos de nosotros/as rondamos por un espacio que nos es cada vez más ajeno y al cual tenemos menos capacidad de transformar. Como señalaba Milton Santos a propósito de la globalización; "as ações são cada vez mais estranhas aos fins próprios do homem e do lugar" (Santos, 1994: 51).

Aquí radica un aporte clave para nuestra profesión, ya que esta reflexión nos posibilita comprender la íntima relación entre las desigualdades económicas y sociales con las diferentes posibilidades que tienen los grupos y clases sociales de transformar y apropiarse del espacio. En el caso del Trabajo Social, su actuación se caracteriza por la relación con aquellos sujetos a los que sistemáticamente se les ha negado dicha posibilidad de producción del espacio que habitan (lo que en clave urbana se ha transformado en reivindicaciones tales como el derecho a la ciudad).

Esta constatación sobre la conformación del espacio, nos remite a una característica levantada con fuerza desde las teorías críticas, esto es, el necesario reconocimiento de la condición política del espacio (razón por la cual comenzamos este apartado con la certera cita de Léfébvre). En un contexto actual marcado por la pérdida de derechos y territorios, cuestionar la neutralidad aparente de nuestro espacio nos parece una tarea fundamental del pensamiento crítico y, en particular, para los/as trabajadores/as sociales que nos situamos desde esta perspectiva.

Uno de los procesos centrales que afectan la capacidad de transformación del espacio en nuestras sociedades, son las consecuencias del capitalismo en su producción. Si bien la dominación del espacio ha sido un elemento clave para la expansión del capitalismo desde su origen, Léfèbvre sostiene que en su crecimiento co- 
mo modo de producción hemos pasado desde la producción en el espacio a la producción del espacio. Esto se evidencia en el protagonismo que ha adquirido dentro de este modo de producción "todo lo relacionado con el espacio; por ejemplo, la construcción, la urbanización, las inversiones en este terreno, la venta y la compra del espacio como totalidad y la especulación, por supuesto" (Léfèbvre, 1974: 220). Es por esto que el autor afirma que el capitalismo ya no se apoya solamente sobre las empresas y el mercado, sino que refuerza su apoyo sobre el espacio para propiciar la acumulación; un lugar de observación privilegiada de cómo se relaciona el capital con el espacio lo encontramos en la conformación de nuestras ciudades latinoamericanas de las últimas décadas. Sobre aquello versa la siguiente sección.

\section{Transformaciones socio-espaciales y ciudades latinoamericanas}

Dentro del debate sobre el espacio presentado anteriormente, vemos a la ciudad como expresión de un espacio dominado y apropiado, atravesado profundamente por los procesos descritos en el apartado anterior. En esa dirección argumentativa, podríamos decir que la ciudad es producto y producción de las relaciones sociales que se generan al interior de ella. Muestra de esto, es que en ciudades como Santiago de Chile vemos las huellas de miles de pobladores que a lo largo de la historia de la ciudad resistieron (y resisten) a la planificación urbana que los negaba, desde el Alarife Gamboa en los tiempos de colonialismo español, pasando por Vicuña Mackenna hasta llegar a la política urbana de 1979. Todo ello, al mismo tiempo que podemos apreciar las marcas de la desigualdad del Neoliberalismo chileno en la ciudad. Muestra de esto es que el área central de Santiago ha experimentado un crecimiento explosivo entre los años 2000 y 2010 del precio de viviendas de mayor costo y con menor superficie, lo que ha excluido paulatinamente a las familias más numerosas y con menores ingresos del centro de la ciudad (LópezMorales, 2013). 
Si continuamos con la reflexión de Léfèbvre que hacíamos en el apartado anterior, donde sosteníamos que "es el espacio y por el espacio donde se produce la reproducción de las relaciones de producción" (Léfèbvre, 1974: 223), podríamos concordar en que la ciudad -en tanto espacio- se ha convertido en uno de los lugares privilegiados para dicha reproducción. Y esto no sólo por la consolidación de las llamadas ciudades globales que analiza Saskia Sassen, las cuales concentran flujos de capital, personas e información, sino también en ciudades de menor jerarquía global, como es el caso de las ciudades del Sur. Sólo como muestra de esto, Davis señala que en la década de los ochenta, cuando el Fondo Monetario Internacional y el Banco Mundial utilizaron la palanca de la deuda para reestructurar las economías de la mayor parte del Tercer Mundo, fue un periodo en que las “... áreas urbanas híper degradadas se convirtieron en un futuro implacable, no sólo para los migrantes pobres rurales, sino también para millones de urbanitas de toda la vida, desplazados o empobrecidos hasta la miseria por la violencia del «ajuste»" (Davis, 2004: 18).

Sumado a esto, en el caso de nuestra Región es un hecho constatado que, en la actualidad, la mayoría de los países de América Latina son cada vez más urbanos, atributos que convierten a las ciudades en observatorios de los fenómenos de la sociedad. En este sentido, la ciudad es un espacio en el que convergen relaciones sociales entre sujetos de diferentes posiciones en la estructura social, pero que están imbricados en una relación de interdependencia (Panez, 2010).

En segundo lugar, es relevante para el Trabajo Social reconocer en su conformación como profesión el desarrollo de la así llamada cuestión urbana. Dicho término aparece con fuerza en los años setenta del siglo anterior en el debate que plantean intelectuales marxistas como Manuel Castells, Henri Léfèbvre y Jean Lojkine que se dedican a pensar los planteamientos de Marx y Engels a la luz de las transformaciones de las ciudades. Aquí, la recuperación de Engels es notable al lograr caracterizar el surgimiento de la cuestión urbana (aunque no la denomine con este concepto). Sus 
análisis en "La situación de la clase obrera en Inglaterra" (1845) son particularmente valiosos, ya que en esta obra Engels explicita la relación existente entre las problemáticas que vivían los trabajadores de las ciudades industriales de Inglaterra y la conformación y consolidación del capitalismo en este país. Desde estas referencias, entenderemos la cuestión urbana como aquel conjunto de problemáticas articuladas de las ciudades y que poseen una raíz estructural en las contradicciones del modo de producción capitalista en la ciudad (Léfèbvre, 1999); problemáticas que eran asumidas como situaciones aisladas o explicadas desde matrices piscologizantes, bajo formas específicas tales como los problemas habitacionales y sanitarios de la población pobre de las ciudades (Maguiña y Manrique, 1985).

En ese sentido, la ciudad y el importante caudal de discusiones teóricas que ha suscitado su debate desde el clásico de Engels de 1845 hasta nuestros días, representan un amplio abanico de posibilidades para que desde el Trabajo Social se nutra de elementos críticos para su reflexión y acción profesional. Si pensamos en el panorama actual de nuestras ciudades: ¿cuáles son las principales transformaciones que han afectado a las ciudades de América Latina? Desde nuestra perspectiva, las ciudades latinoamericanas han sido impactadas por cambios globales a través de tres procesos que esbozamos a continuación y que ya han sido parcialmente abordados en otros trabajos de los mismos autores.

\section{Nuevas formas de aglomeración urbana}

Hablando particularmente de las repercusiones en las ciudades y sus tendencias de aglomeración, De Mattos desmitifica los análisis que afirmaban que la reestructuración productiva de las últimas décadas llevaba a procesos de estancamiento de la expansión metropolitana y una mejor distribución territorial productivo-demográfica. Por el contrario, señala que actualmente "la realidad observable se ha encargado de demostrar que la concentración en grandes aglomeraciones urbanas permanece como rasgo destacado del nuevo escenario" (De Mattos, 2001, p. 18). 
De esta forma, podemos decir que, si bien los procesos de concentración de capital en el espacio de las grandes ciudades se mantienen, la diferencia radica en la forma en que se organiza el espacio urbano y sus habitantes. En las grandes urbes latinoamericanas, paradójicamente a los intentos por atraer las inversiones extranjeras, viene desarrollándose en los países de América Latina (y en los países del Sur en general), procesos de concentración poblacional que no necesariamente se incrementan al ritmo del crecimiento económico de esas ciudades. En el caso chileno, esta tendencia queda en evidencia al revisar la distribución de campamentos (asentamientos precarios) a lo largo del territorio, en donde "un 73 por ciento de los campamentos se encuentran al interior o cercanos a las ciudades más grandes del país" (Sabatini y Brain, 2008: 12).

\section{Protagonismo del mercado inmobiliario urbano}

El mercado inmobiliario como impulsor del modo de producción actual en las ciudades, ha llevado a que el suelo asuma cada vez más importancia en la generación de valor. En la actualidad, esta importancia se plasma en dos características.

En primer lugar, una de las principales características de la transformación urbana es la acelerada demanda de expansión de suelo urbano, la que convierten a las empresas inmobiliarias y de la construcción en actores preponderantes en la toma de decisiones que impactan el crecimiento de la ciudad. Este sector presiona con fuerza para la mantención y creación de normativas, la modificación de planes reguladores de ciudades, la expansión territorial en la provisión de servicios públicos en la ciudad, etc. Lo anterior genera un contexto donde el mercado inmobiliario ha podido aprovechar la especulación de los suelos (comprar suelo barato -algunas veces agrícola- y luego otorgarle valor acogiendo la demanda habitacional).

La segunda característica tiene que ver con la fuerte demanda por el suelo urbano existente en espacios de la ciudad que han sido revalorizados atrayendo a un nuevo segmento de la población. 
Un ejemplo emblemático de este proceso, en el caso de Chile, es la ciudad de Valparaíso, reconocida como patrimonio material e inmaterial por la UNESCO, lo que ha convertido algunos de los puntos de la ciudad en atractivos inmobiliarios para sectores con mayor poder adquisitivo proveniente de otros lugares de la ciudad, e incluso, de otras ciudades del país (Santiago principalmente), provocando un proceso de gentrificación donde los antiguos residentes son desplazados por estos nuevos habitantes (Inzulza y Galleguillos, 2014).

\section{Gestión urbana emprendedora}

Lo que señalamos anteriormente sobre el mercado inmobiliario acontece con la activa participación del Estado, el cual modificó fuertemente su postura en la producción del espacio urbano, caracterizándose, en algunos casos, por su ausencia en la regulación territorial, mientras que, en otros casos, lejos de omitir acciones, muchos Estados latinoamericanos han sido enérgicos en desplegar acciones para flexibilizar normativas en favor de la actividad inmobiliaria, tales como la aplicación de cambios de uso de suelo para la construcción de nuevos proyectos comerciales y habitacionales en las ciudades.

Para entender esta transformación en la acción estatal, David Harvey sitúa como contexto, las modificaciones producidas a partir de la crisis económica de 1973 y los cambios en las orientaciones del gobierno en las ciudades, en donde la capacidad declinante del Estado-Nación de controlar los flujos financieros de las empresas multinacionales ha llevado a que "la inversión asuma cada vez más la forma de negociación entre el capital financiero internacional y los poderes locales" (Harvey, 2006: 168). En este contexto, surge lo que el geógrafo inglés llama "Emprendimiento Urbano" que tiene como uno de sus elementos principales la noción "de asociación Público-Privada, en que la iniciativa tradicional local se integra con el uso de los poderes gubernamentales locales, buscando y atrayendo fuentes externas de financiamiento y nue- 
vas inversiones directas o nuevas fuentes de empleo" (Harvey, 2006: 172).

De esta forma, atraer la inversión se convierte en una de las principales prioridades de la gestión de la ciudad, bajo la cual están sometidas otras necesidades, o bien, se entiende que las otras prioridades aparecen como consecuencia de este fin mayor. Un ejemplo de esta lógica es el auge de la gestión de megaeventos en las ciudades, donde Rio de Janeiro ha sido muestra de cómo la realización de eventos deportivos sirve como justificación para acciones que pueden incluir desde remociones masivas de moradores en áreas de interés inmobiliario, hasta la intervención militar en favelas y privatizaciones en la gestión de la salud y la educación municipal. Las autoridades están convencidas de cambiarle la cara a la llamada cidade maravilhosa a costa de una acción estatal que favorezca la obtención de grandes lucros privados en la ciudad, lo que -según se argumenta- otorgaría beneficios parciales a la población más pobre (Cardoso, 2013).

En el contexto de las transformaciones comandadas por los intereses del capital que han impactado a las ciudades latinoamericanas, somos testigos de la degradación de las condiciones de vida de miles de habitantes que se encuentran confinados y concentrados en territorios determinados de nuestras urbes. Este proceso, debido a la relevancia que adquiere para el análisis de este artículo, será profundizado en el próximo apartado.

\section{Territorios de relegación urbana y marginalidad avanzada}

¿Cómo denominar aquellos lugares donde se aglomera la pobreza urbana? Esta pregunta representa una discusión central para las intervenciones sociales en las que actúan trabajadores/as sociales en ciudades de todo el continente. Lo anterior cobra relevancia en un contexto de irrupción de modos enunciativos que han pretendido sustituir a categorías tradicionales (por ejemplo, poblaciones en Chile, favelas en Brasil, villas en Argentina), colocando en su reemplazo conceptos que tienden a su despolitización y fragmen- 
tación explicativa: la proliferación de los barrios responde a esta tendencia ${ }^{5}$.

En ese sentido, consideramos que para hacer frente a los discursos que predominan en las políticas sociales, es muy provocativo y fecundo el análisis que ofrece la obra de Loïc Wacquant. Desde nuestra apropiación crítica de dicho análisis, consideramos que es posible discutir los eufemísticos términos que nos brinda la semántica dominante -barrios vulnerables, sectores marginales-humildes-desaventajados-pobres, comunidades de bajos ingresos, por citar algunos ejemplos-, para proponer el de territorios de relegación urbana, el cual no agota el debate, pero sí lo profundiza críticamente.

¿Es lo mismo hablar de barrios que de territorios de relegación urbana? ¿Es acaso indiferente el modo en que nos referimos a dichos territorios? ¿Qué implicancias posee uno u otro modo de enunciación para la política social en general y la intervención social en particular? Las diferencias son abismantes y profundizar en ellas es una tarea central, considerando que dependiendo del referencial utilizado se desprenderán derivaciones teóricas y ético-políticas de gran relevancia, las que determinarán el tipo de intervención social resultante y, por tanto, el abanico de posibilidades de actuación profesional que los/as trabajadoras/es sociales involucrados en esos procesos de intervención desarrollarán.

La noción de territorios de relegación urbana intenta referirse a aquellas zonas de la ciudad, generalmente en sus periferias, donde se concentran la ausencia y/o precariedad de servicios básicos, una población económicamente activa mayoritariamente desempleada o empleada precariamente, agudos niveles de pobreza, estigmatización simbólica, entre otros elementos que en los párrafos

Los programas Quiero Mi Barrio (Chile), Favela Bairro (Brasil), Programa de Mejoramiento de Barrios-PROMEBA (Argentina), Barrios de Verdad (Bolivia), Mi Barrio Lindo (Quito, Ecuador), Mejoramiento de Barrios (Paraguay), Por mi Barrio (Honduras), Programa Mejoramiento Integral de Barrios (Colombia, Perú), Barrio Seguro (Guatemala), por mencionar sólo algunos, dan cuenta de ese movimiento. 
siguientes desarrollaremos. En ese sentido, si bien no existe una referencia específica donde el autor establezca qué es un territorio de relegación urbana (a modo de definición), éste se encuentra estrechamente vinculado con el concepto de marginalidad avanza$d a$, que nos ofrece lúcidas orientaciones para comprender la forma en que, en el actual contexto de conformación de ciudades antes descrito, emergen estos territorios y cuáles son sus principales características.

En primer lugar, el concepto comienza por darle un papel central a las transformaciones que, a partir de la crisis de 1973 antes citada, se han venido sucediendo en el modo de organización del trabajo. En ese sentido, los procesos de flexibilización producto de las prerrogativas del patrón de acumulación capitalista (Harvey, 1993), exigieron la máxima desregulación posible de los mercados laborales provocando la erosión de la capacidad integradora del trabajo asalariado. Así, fueron activados -sostendrá Wacquanttodos los caminos posibles "para evitar los efectos homogeneizantes del patrón de regulación estatal del trabajo asalariado"; sindicatos y rubros tradicionales de producción dieron lugar a las precarias condiciones de los trabajos a domicilio, los tele-trabajos y la institucionalización del trabajo permanentemente temporario: "cada uno de los elementos de seguridad concedidos sobre el contrato social fordista-keynesiano fue debilitado o está siendo atacado" (Wacquant, 2001: 171).

En segundo lugar, existe una desconexión funcional entre la macroeconomía y la suerte que corren los habitantes de las zonas de relegación urbana, donde las fases de expansión de empleo y consumo no tienen efectos duraderos sobre ellos, efectos que -de otro lado- sí se sienten con fuerza cuando hay fases de menor crecimiento económico. La irrenunciable vocación de acumulación de la clase capitalista alarga inapelablemente la eterna espera por el chorreo (trickle down effect) profetizada por el catequismo neoliberal; de allí que podemos comprender el hecho de que, cuando hay tiempos de bonanzas, las mayores utilidades -al contrario de lo que se prometía- no se distribuyen hacia el resto 
de la población, sino que van a abultar las riquezas de quienes las acumulan.

En tercer lugar -y este es quizás el que más directamente se relaciona con el concepto mismo de territorios de relegación urbana-, emerge la concentración de los pobres urbanos en sectores simbólica y territorialmente marcados y cada vez más aislados, que atraen la atención desmedidamente negativa de la prensa, los políticos y los dirigentes del Estado, lugares donde se congregan e infeccionan los problemas sociales, lugares que son “...vistos por personas de dentro y de afuera como purgatorios sociales, infiernos urbanos donde sólo los desechos de la sociedad aceptan habitar" (Wacquant, 2001: 172). Son lugares donde la presión externa, la ausencia de servicios sociales, la pésima calidad de vida y la abundancia de complejas problemáticas atentan contra cualquier tipo de sentimiento de pertenencia, donde sus habitantes, para conseguir empleos, falsean la dirección de sus casas, lugares donde existe un constante intento de distanciarse -social y simbólicamente- del lugar donde residen: lugares que crean "un indivisible sentimiento de vergüenza y culpa cuyo peso no reconocido pervierte las relaciones humanas" (Pétonnet, 1982; Wacquant, 2001: 173) .

Lo anterior determina que, en un cuarto lugar, dichos sectores sufran de una alienación territorial, esto es, "la pérdida de un lugar con el cual las poblaciones urbanas marginalizadas se identifiquen y en los cuales se sientan seguras" (Wacquant, 2001: 173). Y es que la reconfiguración actual del capitalismo no involucra apenas dimensiones económicas, sino que implica una "transformación muy importante en la organización y la experiencia del propio espacio" (Harvey, 1989; Wacquant, 2011: 173).

En el texto referido, Wacquant cita el testimonio de un joven que, en el marco de la investigación realizada por Pétonnet en una banlieu francesa, afirma justamente sentirse avergonzado del lugar donde vive, el cual es negado. Sobre eso, Wacquant agrega testimonios de sus propias investigaciones en el gueto negro de Chicago, a lo que sumamos nuestra experiencia como asistentes sociales, en El Castillo, comuna de La Pintana (Santiago de Chile), donde era también común escuchar a vecinos y vecinas afirmar: "nosotros no somos de El Castillo, los de El Castillo son los de más allá...” 
En estos sectores, fuertemente precarizados, los lazos de solidaridad se vuelven cada vez más difíciles de sustentar; en momentos de crisis económica, en los antiguos sectores marginalizados, aún existía la posibilidad de que sus miembros al perder sus empleos, pudieran auxiliar a la colectividad. Actualmente, dichas áreas...

...se vienen virtualmente vaciando de sus medios de sustento colectivo y los vínculos con el trabajo asalariado externo son drásticamente disminuidos por la desproletarización total de grandes segmentos de la población local (...) Para sobrevivir, deben recurrir a estrategias individuales de 'autoabastecimiento', 'trabajo clandestino' y comercio informal (Wacquant, 2001: 175).

Finalmente, y ya se deduce del punto anterior, una sustancial diferencia de los actuales contextos de marginalidad con las décadas anteriores dice relación con un proceso de descomposición de clase en vez de uno que apunte a su formación o consolidación: "La ausencia de un lenguaje común que los unifique simbólicamente acentúa la dispersión y la fragmentación de los nuevos pobres urbanos" (Wacquant, 2001: 175).

En estos contextos de erosión forzosa de lazos sociales, de barbarización extrema de las condiciones materiales de vida y de un ataque simbólico inmisericorde contra quienes habitan un determinado territorio, se levantan programas sociales con objetivos, por ejemplo, de promover la identidad barrial, la solidaridad e integración comunitaria entre vecinos/as y los sentimientos de pertenencia. En aquellos programas se emplean, masivamente, trabajadores y trabajadoras sociales que probablemente vivencian, en la práctica cotidiana de su acción profesional, las situaciones acá descritas. Es entonces cuando nos preguntamos... ¿cómo explican, ¿cómo leen esas situaciones? ¿De qué manera reflexionan sobre ellas, cómo las teorizan?

A las características de la marginalidad avanzada antes descrita, es necesario adicionar otros elementos que vuelven aún más complejo el escenario. A la relegación urbana, el confinamiento es- 
pacial, la estigmatización social, el desprecio cotidiano desde los otros sectores de la sociedad, la criminalización publicitada por los medios de comunicación, los parias urbanos atestiguan como ninguna otra clase social la emergencia de una fase penal del Estado. Se trata del reemplazo de la providencia por la penitencia, que los vuelve receptores de las acciones del Estado, pero no de aquellas acciones destinadas a fortalecer su condición de sujetos de derecho, sino de aquellas destinadas a disciplinarlos y reprimir sus conductas desviadas: "La atrofia del Estado Social y la hipertrofia del Estado Penal son dos transformaciones complementarias y correlativas que forman parte de la institución de un nuevo gobierno de la miseria" (Wacquant, 2001: 198).

Si bien los análisis que el autor presenta en "Las cárceles de la miseria" (Wacquant, 2003) y "Castigar a los pobres" (Wacquant, 2002) responden a la realidad europea y estadounidense, existen elementos de nuestra cuestión urbana que podemos leer a partir de ellos. En este sentido, el abandono del Estado Providencia (que en América Latina nunca tuvo un desarrollo como en Europa) y su reemplazo por un Estado de Penitencia, nos "anuncia la aparición de una nueva forma de gobernar la miseria, que une la mano invisible del mercado de trabajo descalificado y desregulado con el puño de hierro de un aparato penal intrusivo y omnipresente" (Wacquant, 2003).

En nuestras ciudades crecen estos territorios, la sociedad misma los va creando y, sin embargo, esta última se declara temerosa de ellos y procura enérgicamente los modos de mantenerlos alejados. Esta forma conservadora de asumir a los pobres urbanos como clases no pertenecientes a la ciudad, omiten lo mucho que dependen nuestras ciudades de dichos sectores: ¿De dónde obtiene mano de obra barata el emprendedor capitalista? ¿Dónde arrojan sus escombros y la basura las grandes empresas constructoras sino en vertederos (legales e ilegales) ubicados en las zonas periféricas de las ciudades? ¿Dónde son construidas las cárceles, plantas de tratamientos de desechos y diversos equipamientos urbanos que toda la ciudad necesita pero que sólo los pobres deben aceptar co- 
mo vecinos? ¿Dónde compra drogas la juventud de las otras clases sociales, de los otros sectores de la ciudad?

Es en estos territorios simbólica y materialmente relegados de la ciudad donde cientos de asistentes/trabajadores sociales a lo largo del continente desarrollan sus intervenciones sociales en la búsqueda, tan honesta como -muchas veces- infructuosa, de mejorar las condiciones de vida de los habitantes de dichos espacios marginalizados en el contexto urbano. ¿A partir de qué elementos explican la situación que enfrentan día a día? Para esbozar un camino preliminar de aproximación a dicha pregunta, efectuamos un ejercicio indagatorio centrado en dos aspectos que nos puedan dar algunas pistas: los programas de estudios (mallas curriculares) y las ponencias/trabajos presentados en encuentros latinoamericanos de Trabajo Social en los últimos años. A continuación, los resultados de dicha revisión.

\section{Rastreando el debate actual en Trabajo Social}

Para indagar sobre lo primero, es decir, la formación profesional, revisamos los planes de estudios (mallas curriculares) de 73 programas de pregrado (graduación/grado) en Trabajo/Servicio Social de 18 países en América Latina: Argentina (10), Bolivia (5), Brasil (18), Chile (13), Colombia (5), Costa Rica (1), Ecuador (3), Guatemala (1), Honduras (1), México (4), Nicaragua (1), Panamá (1), Paraguay (1), Perú (2), Puerto Rico (2), República Dominicana (1), Uruguay (2), Venezuela (2) ${ }^{7}$. Buscamos la presencia de cursos (materias/disciplinas) ${ }^{8}$ al interior de los planes de estudios en

Los criterios de selección para este ejercicio indagatorio -que, vale aclarar, no pretende se representativo, apenas una primera aproximación- fueron: (i) programas que tuvieran posgrados (con lo cual intentamos asegurarnos de considerar a aquellos que poseen un mayor desarrollo en el debate académico dentro de sus países), (ii) los programas en los que sus académicos participen de las actividades profesionales de ALAEITS y además que participen con ponencias en encuentros internacionales, (iii) los que poseyeran, como mínimo, un sitio web de sus programas. En el ANEXO se detallan las Universidades indagadas por país.

8 Programas o Planes de Estudios es igual a Mallas Curriculares, mientras que el pregrado, grado y graduación se refieren al proceso de formación inicial de los asistentes/trabajadores sociales, en programas que en promedio van de los 4 a 5 
cuyo título estuviese contenido uno de los siguientes cinco conceptos: ciudad-territorio-marginalidad-urbano/a-espacio. Al excluir de la muestra los 18 programas que no presentaron información para confirmar/descartar la existencia de los cursos procurados, llegamos a un total de 55 programas. De dichos programas, 40 "NO" presentaron ninguno de los conceptos buscados (73\%), mientras que 15 de ellos "SÎ" presentaron (27\%).

Gráfico 1:

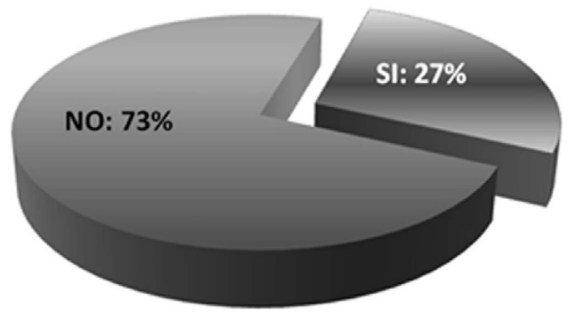

En el Plan de Estudios del programa... ¿existe al menos un curso/materia que contenga en su título algunos de los siguientes conceptos: ciudad-territorio-marginalidad-urbano/a-espacio?

Fuente: Elaboración propia

Los 15 programas donde "SÍ" registramos la presencia de, a lo menos, uno de los conceptos buscados, fueron: urbano/a (8), territorio (6) y ciudad (1), pertenecientes a Argentina, Bolivia, Brasil, Chile, Colombia, Ecuador, México, Uruguay y Venezuela; es decir, ni marginalidad ni espacio aparecen en la revisión de los cientos de cursos/ materias ofrecidos en esas mallas curriculares. Ahora bien, a partir de lo anterior no sería posible decir que en la formación académica de los/as asistentes sociales no aborden las cuestiones aquí tratadas, pues comprendemos que el hecho de que no estén explicitadas en un título de una materia/curso, no quiere decir que no se hable del tema. Sin embargo, la pregunta es... ¿Cómo se habla? ¿Al interior de qué cursos? ¿Desde qué referenciales teóricos? ¿En base a qué 
autores? ¿Cuál será la hondura conceptual del debate que estamos ofreciendo a los futuros asistentes sociales en esta temática? Son preguntas que nos podrían dar luces acerca del cómo nos relacionamos desde el Trabajo Social con este debate y que, al menos en este trabajo, deberán quedar en estado de formulación.

Tabla 1: Frecuencia de ponencias en cuyo título existe alguno de los 5 conceptos. Fuente: Elaboración propia

\begin{tabular}{|c|c|c|c|}
\hline Encuentro / Congreso / Seminario & CONCEPTO & Total Ponencias & Porcentaje \\
\hline V Encuentro Política Social y TS - Costa Rica 2003 & 0 & 66 & 0,0 \\
\hline ALAETS San José - Costa Rica 2004 & 0 & 83 & 0.0 \\
\hline ALAETS Guayaquil- Ecuador 2009 & 13 & 350 & 3,7 \\
\hline Encuentro Investigación - México 2011 & 1 & 37 & 2,7 \\
\hline ALAEITS Córdoba - Argentina 2012 & 6 & 245 & 2,4 \\
\hline V Encuentro Sur Andino de Trabajo Social - Chile 2012 & 6 & 107 & 5,6 \\
\hline III Congreso Conocimiento - Chile 2013 & 1 & 20 & 5,0 \\
\hline XXVIII Congreso Nacional de Trabajo Social - Argentina 2014 & 6 & 166 & 3,6 \\
\hline XXI Seminario Latinoamericano de Trabajo Social- México 2015 & 11 & 335 & 3,3 \\
\hline
\end{tabular}

En relación a los encuentros de Trabajo Social a nivel latinoamericano, nos centramos en aquellos que disponían de información online sobre sus anales y ponencias, otorgándole relevancia a la revisión de los programas generales de los últimos tres seminarios latinoamericanos de ALAEITS. A pesar de la escasa información disponible, conseguimos revisar más de mil ponencias, agrupadas en 9 diferentes encuentros, desde el 2003 hasta el 2015. Apenas un $3,1 \%$ de ellas contenía, en su título, alguno de los 5 conceptos antes mencionados (columna "CONCEPTO").

Dentro de la escasez evidente de la temática, donde apenas un casi inexistente $3,1 \%$ de ellas abordan, directamente -a juzgar por el título de los trabajos- los contenidos de interés de este artículo, podemos destacar que en el Encuentro Sur Andino realizado en Chile (2012), existió una mesa de trabajo denominada "la dimensión socioespacial de la desigualdad". Es decir, se trata no sólo de una escasa presencia de ponencias/trabajos, sino también de una casi nula presencia de espacios dedicados a discutir investigaciones relacionadas con nuestra temática de interés en este escrito.

Ahora bien, del total de 33 ponencias que explícitamente usan "nuestros" conceptos claves, 11 de ellas pertenecen a investigado- 
res de universidades brasileras, 8 provenientes de Argentina y 7 desde Chile. Es decir, en términos de presencia de trabajos que debaten dichas temáticas al interior de las discusiones que tenemos como Trabajo/Servicio Social latinoamericano, apenas en tres países -con mayor presencia en el Cono Sur- los y las colegas del colectivo profesional dialogan directamente con algunos de los conceptos aquí presentados. ¿Qué ocurrirá con los otros países? ¿No se discutirá acerca del tema?

Por supuesto que una conclusión como esa sería extremadamente apresurada, pues es altamente probable que las temáticas sí sean discutidas, ya sea en otros espacios (en encuentros sobre urbanismo, por ejemplo), ya sea por otras vías de entrada (por ejemplo, infancia y segregación, migraciones, etc.). Pero, a juzgar solamente por estos datos, al interior de la profesión el debate es prácticamente inexistente, lo cual representa un contrasentido gigantesco, atendiendo al hecho factual de que existe una masiva presencia de trabajadores y trabajadoras sociales en los procesos de intervención en dichos territorios.

Por lo anterior, resulta central avanzar en las investigaciones, donde la precariedad de la información disponible es el primer escollo a sortear: es difícil encontrar un listado de todas las escuelas de trabajo social por cada país de América Latina, un sitio web con información de cada una de ellas, menos aún sitios web en los que esté disponible la información acerca de los planes de estudio; y todavía menos aún, descripciones respecto de los contenidos de cada uno de los cursos/materias, que es, en definitiva, el lugar donde por la vía de la revisión de las estructuras de dichos cursos y las bibliografías utilizadas, podríamos hacernos una idea un poco más certera respecto de cómo están siendo abordados -si es que lo están siendo- conceptos como espacio, urbanismo, ciudad, territorio y marginalidad (u otros, a través de los cuales los estudiantes de Trabajo/Servicio Social accedan a las discusiones en este artículo planteadas). Hay que considerar que estamos refiriéndonos a los cursos de graduación/pregrado (formación inicial), pues sabemos que en nivel de posgrados la situación tiende 
a cambiar, presentando una mayor cantidad de núcleos de investigación, por lo que las preguntas que en ese sentido pueden emerger, dicen relación con cómo propiciar un diálogo más fecundo entre ambos niveles (pre y posgrado) de formación. La agenda de investigación, es, en este sentido, tan profusa como urgente y nos permite pensar en una serie de proyecciones para nuestra profesión.

\section{Consideraciones finales: desafíos y proyecciones}

Antes que todo, creemos importante señalar una reflexión necesaria sobre los alcances y límites del ejercicio indagatorio propuesto. En este sentido, la revisión de cientos de cursos en más de 50 planes de estudios en 18 países del continente, como también la revisión de más de mil ponencias de los últimos encuentros de la profesión (2003 en adelante) nos ofrece un primer panorama en el intento de dibujar un mapa latinoamericano acerca del cómo estamos, desde el Trabajo Social, abordando teóricamente aquellas temáticas que son bastante recurrentes en nuestras prácticas profesionales. Y esa es, probablemente, la mayor inquietud que nos instiga a escribir este artículo, pues estamos hablando de un área de intervención masivamente ocupada por asistentes sociales, pero que, sin embargo, posee, a modo de correlato teórico, una discusión que está lejos de ser masiva y profunda. Lo anterior nos plantea varios desafíos.

En primer lugar, desafíos teórico-metodológicos. Nuestra invitación a pensar críticamente la ciudad y los procesos de marginalidad avanzada contenidos en ella, necesariamente pasa por una renovación y complementariedad de las actuales perspectivas teóricas con las que el Trabajo Social interviene en este ámbito. ¿Qué elementos hasta ahora descritos están presentes en las reflexiones cotidianas de los/as miles de trabajadores/as sociales inmersos en estos contextos? Frente a lecturas tradicionales o auto-proclamadas críticas que tienden a análisis abstractos y a-espaciales, creemos importante relevar la importancia de la disputa teórica al interior del colectivo profesional sobre las formas de comprender 
y analizar las problemáticas sociales que suceden en las ciudades que intervenimos, pues no se trata sólo de invitar a pensar la ciudad de manera genérica, sino de reflexionar sobre ella críticamente, reconociendo el carácter conflictivo, ideológico y desigual de la producción del espacio urbano (Léfèbvre, 1976).

En ese sentido, consideramos relevante estar atentos al desarrollo teórico y metodológico (por ejemplo, etnografías urbanas o cartografías sociales) de otras disciplinas que piensan la ciudad (antropología, geografía, sociología, arquitectura, etc.). No para importar irreflexivamente saberes de otras disciplinas, sino para entender sus lógicas de funcionamiento, analizar detenidamente su pertinencia y dilucidar bajo qué condiciones esas herramientas podrían ser útiles. Lo anterior nos abriría posibilidades para desarrollar una reflexión al interior de nuestra disciplina y para una práctica profesional crítica, que esté menos del lado de la reproducción de lógicas discursivas liberales que dominan la política social, y más del lado de los sujetos que viven en los espacios relegados de las ciudades y la justa reivindicación de sus derechos.

En segundo lugar, y en términos de algunos desafíos para nuestra formación académica, consideramos que, si bien ninguna de las dos muestras acá presentadas es representativa, sí, al menos, nos da algunas luces respecto de la preocupación central de este escrito: en el Trabajo Social la reflexión sobre una lectura espacial y la marginalidad urbana no son, todavía, temáticas o áreas con cierto nivel de madurez y autonomía, como sí lo son otras áreas que han conseguido impulsar, al interior de la discusión académica, un desarrollo más amplio.

En base a una reflexión y a un debate más consistente al interior del colectivo profesional, se iniciaron y consolidaron procesos que han venido moldeando sus áreas de reflexión y acción, derivando en núcleos de investigación, especialidades, cursos de post graduación, o, como mínimo (lo que buscamos aquí), cursos en la formación inicial que se dediquen abierta y explícitamente a dichas temáticas. Así, por ejemplo, es posible encontrar en los pla- 
nes de estudio cursos/materias como Trabajo Social y Familia, TS $y$ desarrollo rural, TS $y$ desarrollo de la infancia, TS y pueblos originarios, TS y género, TS y Tercer Sector, entre otros. ¿Podríamos pensar, por ejemplo, en masificar el binomio categorial de Trabajo Social y Cuestión Urbana (u otros afines)?

En tercer lugar, como desafío para los programas sociales, resulta fundamental cuestionarnos: ¿de qué forma nuestros programas sociales se hacen cargo de los procesos descritos en las ciudades? O incluso, ¿en qué medida los programas existentes contribuyen a la reproducción de las condiciones de relegación y marginalidad? En el caso chileno, tenemos la experiencia de una oficialmente exitosa política de vivienda en la década de los ochenta y noventa que, pretendiendo resolver el problema habitacional, agravó la cuestión urbana en las grandes ciudades del país consolidando espacios segregados y estigmatizados (Rodríguez y Sugranyes, 2005). Frente a esto, requerimos como profesión complejizar y densificar nuestras propuestas conceptuales y metodológicas acerca del diseño de los programas, nuestra forma de comprender al sujeto y la problemática que buscamos intervenir, teniendo como principal arma de disputa la solidez teórico-crítica de nuestros planteamientos.

Finalmente, en términos de desafíos ético-políticos, consideramos que hacernos cargo de este déficit de teorías críticas es central para relevar el compromiso del Trabajo Social con la Justicia Social y la lucha contra las desigualdades y abusos propios del sistema capitalista, al lado de las clases que más los padecen. En diferentes ciudades latinoamericanas es posible respirar la desigualdad y abandono territorial de los miles y miles de personas que las habitan. Son ellos y ellas los que nos desafían a esta invitación de ampliar la mirada del Trabajo Social desde sus territorios relegados: sus dolores, rabias, impotencias y esperanzas nos deben orientar para proponer enfoques pensados desde los bordes de la ciudad neoliberal donde buscamos, como profesión, nuestro lugar. 
El desafío implica reconocer la dimensión política de la profesión en las relaciones de fuerzas que se disputan la producción de la ciudad. Desde nuestra posición, para avanzar en esta tarea desde una perspectiva crítica del Trabajo Social, es importante comprender que las transformaciones actuales de las ciudades latinoamericanas están fuertemente ligadas a las configuraciones de la cuestión urbana, entendiendo ésta como una manifestación concreta de la cuestión social dentro del capitalismo contemporáneo (Cabral, Lima y Alves, 2012). Por lo tanto, es tarea central explicitar la dimensión espacial y urbana dentro del debate actual sobre el proyecto ético-político profesional, para dotar de mayores y efectivas herramientas políticas y teóricas el actuar de trabajadoras y trabajadores sociales esparcidos a lo largo y ancho de nuestro continente, tan rico en recursos y posibilidades como pobre en su capacidad de garantizar el ejercicio de derechos de las grandes mayorías. Allí radica nuestra principal tarea.

\section{Referencias bibliográficas}

Cabral, M. F., Lima, L. y Aves, B. (2012). Pesquisa em favelas: contribuições do Núcleo de Pesquisa e Extensão Favela e Cidadania ao debate sobre os desafios postos para o Serviço Social. Revista O Social em Questão, n²7, 133-150.

Cardoso, I. (2013). O papel da Operação Urbana Consorciada do Porto do Rio de Janeiro na estruturação do espaço urbano: uma 'máquina de crescimento urbano'? Revista O Social em Questão, n² 29, 69-100.

De Mattos, C. (2001). Movimientos del capital y expansión metropolitana en las economías emergentes latinoamericanas. Revista de Estudios Regionales, V. II, n. 60, p. 15-43.

Escobar, A. (2014). Sentipensar con la Tierra. Nuevas lecturas sobre desarrollo, territorio y diferencia. Ediciones UNAULA, Colombia.

Harvey, D. (1993). A condição pós-moderna. São Paulo, Ed. Loyola. (2006). A Produção Capitalista do Espaço. Editorial Anna Blume.

Hiernaux, D. y Lindón, A. (2008). Compartir el espacio: Encuentros y desencuentros de las Ciencias Sociales y la Geografía Humana. En "X Años de Estudios Sociales en la Universidad Autónoma Metropolitana Iztapalapa”, México. 
Iamamoto, M. (2003). A Questão Social no Capitalismo. Revista Praia Vermelha $\mathrm{N}^{\circ} 8$, p.56-83.

Inzulza, J. y Galleguillos, X. (2014). Latino-gentrificación y polarización: transformaciones socioespaciales en barrios pericentrales y periféricos de Santiago, Chile. Revista de Geografía Norte Grande, n58, p. 135-159.

Léfèbvre, H. (1974). La producción del espacio. Papers: Revista de sociología, 3, p. 219-229.

(1976). Espacio y Política. El Derecho a la Ciudad II. Ediciones Península. Barcelona.

(1999). A Cidade do Capital. DP\&A Editora, Rio de Janeiro.

Manguiña, A. y Manrique, M. (1985). Pobreza Urbana y Políticas de Vivienda: El Trabajo Social en la problemática habitacional. Ediciones CELATS.

Panez, A. (2010). La disputa por la ciudad: la importancia de una perspectiva urbana crítica en Trabajo Social. Revista O Social em Questão, Año XIII, n²4, P. 55-68.

Rodríguez, A. y Sugranyes, A. (2005). Los con techo. Un desafío para la política de vivienda social. Ediciones SUR, Santiago de Chile.

Sabatini, F. y Brain, I. (2008). La segregación, los guetos y la integración social urbana: Mitos y Claves. Revista de Estudios Urbano Regionales, EURE No 103, p. 5-26.

Santos, M. (1994). A natureza do espaço. Editora da Universidade de São Paulo.

Wacquant, L. (2004). Las cárceles de la miseria. Buenos Aires. Editora Manantial.

(2013). Marginalidad, etnicidad y penalidad en la ciudad neoliberal: una cartografía analítica. Ethnic \& $R a-$ cial Studies, Symposium, 2013 [Traducción de Luján Vega e Ignacio González]. Disponible en http://loicWacquant. net/assets/Papers/Recent-Papers/Wacquant-Marginalidad-2014.pdf. Accedido en: 18 jun. 2015.

(2001). Os Condenados da Cidade: estudos sobre marginalidade avançada. Rio de Janeiro. Editora Revan.

(2002). Punir os Pobres. A Nova Gestão da Pobreza nos Estados Unidos. Rio de Janeiro. REVAN/Instituto Carioca de Criminologia. 


\section{ANEXO:}

\section{Listado de Universidades indagadas por país}

\begin{tabular}{|l|l|}
\hline País & Universidad \\
\hline Argentina & Universidad Nacional de Córdoba \\
\hline Argentina & Universidad Nacional de la Plata \\
\hline Argentina & Universidad Nacional de Rosario \\
\hline Argentina & Universidad Nacional de Entre Ríos \\
\hline Argentina & UBA \\
\hline Argentina & Universidad Nacional de Cuyo \\
\hline Argentina & Universidad Nacional del Litoral \\
\hline Argentina & Universidad Nacional de Luján \\
\hline Argentina & Universidad Nacional de Misiones \\
\hline Argentina & Universidad Nacional de Catamarca \\
\hline Bolivia & UBI \\
\hline Bolivia & UNI MAYOR SAN SIMON \\
\hline Bolivia & Universidad Mayor de San Andrés \\
\hline Bolivia & U. Autónoma Tomás Frías \\
\hline Bolivia & U. Autónoma Gabriel René Moreno \\
\hline Brasil & PUC Sao Paulo \\
\hline Brasil & PUC Rio de Janeiro \\
\hline Brasil & Universidade de Estado do Río de Janeiro \\
\hline Brasil & PUC Rio Grande Do Sul \\
\hline Brasil & Universidade Federal Alagoas \\
\hline Brasil & Universidade Federal de Juiz de Fora \\
\hline Brasil & Universidade Federal de Santa Catarina \\
\hline Brasil & Universidade Federal do Piaú́ \\
\hline Brasil & Universidade do Pará \\
\hline Brasil & Universidad Federal de Pernambuco \\
\hline Brasil & Universidade Federal do Maranhão \\
\hline Brasil & Universidade Nacional de Brasilia \\
\hline Brasil & Universidade Estadual Paulista/ Franca \\
\hline Brasil & Universidade Federal Fluminense \\
\hline Brasil & Universidade Federal de Espíritu Santo \\
\hline Brasil & Universidade Estadual de Londrina \\
\hline Brasil & Universidade Federal de Río de Janeiro \\
\hline Brasil & Universidade Federal do Rio G. do Norte \\
\hline Chile & U. Academia Humanismo Cristiano \\
\hline Chile & Universidad Autónoma de Chile \\
\hline Chile & Universidad de Concepción \\
\hline Chile & Universidad del Bíobío \\
\hline
\end{tabular}


El debate sobre la cuestión urbana y el Trabajo Social: contribuciones críticas para pensar la profesión en territorios de relegación urbana / Orellana y Panez

\begin{tabular}{|l|l|}
\hline Chile & Universidad de Magallanes \\
\hline Chile & Universidad Católica Silva Henríquez \\
\hline Chile & Universidad Santo Tomás \\
\hline Chile & Universidad Alberto Hurtado \\
\hline Chile & Universidad San Sebastián \\
\hline Chile & Universidad de Valparaíso \\
\hline Chile & Pontificia Universidad Católica de Chile \\
\hline Chile & Universidad de Chile \\
\hline Chile & Universidad ARCIS \\
\hline Colombia & Universidad Nacional de Colombia \\
\hline Colombia & Universidad del Valle \\
\hline Colombia & Universidad de Antioquia \\
\hline Colombia & Universidad de Externado \\
\hline Colombia & Universidad de Caldas \\
\hline Costa Rica & Universidad de Costa Rica \\
\hline Ecuador & Universidad Laica Eloy Alfaro \\
\hline Ecuador & Pontificia Universidad Católica de Ecuador \\
\hline Ecuador & Universidad Central de Ecuador \\
\hline Guatemala & Universidad de San Carlos de Guatemala \\
\hline Honduras & Universidad Nacional Autónoma de Honduras \\
\hline México & UNAM \\
\hline México & Universidad Autónoma de Nuevo León \\
\hline México & Universidad Guadalajara \\
\hline México & Universidad Autónoma de Sinaloa \\
\hline Nicaragua & Universidad Nacional de Nicaragua \\
\hline Panamá & Universidad de Panamá \\
\hline Paraguay & Universidad Nacional de Asunción \\
\hline Perú & Pontificia Universidad Católica del Perú \\
\hline Perú & Universidad N. Mayor de San Marcos \\
\hline Pto. Rico & PUC Puerto Rico \\
\hline Pto. Rico & Universidad de Puerto Rico \\
\hline Rep. Dom & Universidad Autónoma de Santo Domingo \\
\hline Uruguay & Universidad de La República \\
\hline Uruguay & Universidad Católica de Uruguay \\
\hline Venezuela & Universidad Central de Venezuela (UCV) \\
\hline Venezuela & Universidad de Zulia \\
\hline
\end{tabular}

